\title{
Modelagem e simulação de pinça mecânica sensoreada
}

\author{
Marcos Felipe Saito
}

\begin{abstract}
Resumo - O projeto baseia-se na modelagem, simulação e controle, feita no software MATLAB, de uma pinça mecânica acionada por um motor com o intuito de segurar um objeto de baixa resistência à pressões superficiais, portanto feito com controle preciso sob a força à ser aplicada no objeto. O projeto é o estudo de um modelo simples de apenas um grau de liberdade, mas é a base dos modelos de prótese mecânica atualmente, dotados de mais graus de liberdade.
\end{abstract}

Palavras-chave: Próteses ortopédicas; Biomecânica; Robótica; Modelagem; Controle; Sensores biomédicos

\section{Introdução}

Por toda a História há registros do uso de próteses,com objetivo de substituir um membro ou parte do corpo. Um dos registros mais antigos é de um dedão do pé feito de madeira, encontrado no Egito, datado do período do Egito Antigo. As próteses mais antigas e mais comuns possuem a simples função de repor uma parte ausente do corpo, não permitindo a mesma funcionalidade do corpo humano.

A prótese robótica é desenvolvida na função de substituir os membros ou até amelhorar a capacidade do usuário, como proposto pelos projetos da DARPA da criação de membros robóticos comandados pelo cérebro, feito para veteranos de guerra americanos amputados.

O objetivo da prótese robótica é dar funcionalidade à prótese, com a intenção de se assemelhar aos membros biológicos, tanto nos movimentos quanto na força aplicada e o feedback de cada ação. A prótese robótica possui três componentes básicos em seu funcionamento:

- O biosensor, que detecta sinais do sistema nervoso e/ou muscular da pessoa; e os transmite para um controlador. Além disso processa o feedback da prótese e do atuador, enviando para o controlador. A prótese mioelétrica usa os sinais eletromiográficos do membro residual para o controle da prótese. A eletromiografia, por sua vez, é técnica de analisar os sinais emitidos pelos músculos esqueléticos, variando de acordo com a diferença de potencial efetivada, e dela se utilizar. No caso, o sinal é captado no membro residual e transmitido ao controlador.

- O controlador envia os comandos desejados da pessoa para a atuador, e também interpreta o feedback dos biosensores e sensores mecânicos. O controlador monitora e controla os movimentos da prótese.

Versão inicial submetida em 03 de agosto de 2017. Versão final aceita em 16 de novembro de 2017. Publicado em 16 de novembro de 2017. Digital Object Identifier: 10.11606/issn.2526-8260.mecatrone.2017.135085 
- O atuador, que imita as ações do músculo em questão, produzindo a força e o movimento requerido, segundo comando do controlador.

O biosensor envolve a captação e interpretação dos comandos biológicos, enquanto o controlador e o atuador atuam na parte da mecânica e do controle da prótese. Na prótese são utilizados sensores mecânicos e elétricos necessários para a detecção dos movimentos realizados e a força aplicada sobre um objeto. A interface de interação entre o usuário e a prótese não será tratada neste projeto, limitando-se apenas a analisar a ação mecânica do mecanismo, tratando o sinal recebido como um sinal elétrico qualquer. O estudo do processamento é estudado na Faculdade de Cornell de medicina, sendo uma pesquisa interdisciplinar entre a engenharia e a medicina. O sinal processado final é apenas um sinal elétrico possível de ser analisado e utilizado.

O projeto envolve a modelagem de uma pinça mecânica dotada de sensores na sua extremidade, para a realização do controle. Apesar do envolvido ser simples, com apenas um grau de liberdade, ele engloba todos os conceitos básicos requeridos necessários para o desenvolvimento de projetos mais complexos de maior graus de liberdade, como mostrados nas figuras abaixo, que fornecem ao usuário uma grande quantidade de movimentos, semelhantes às praticadas pelos membros do ser humano ou de animais. Observa-se a utilização, por toda a extensão das próteses, de partes com o movimento de pinça, como a ação dos dedos, pulso, flexão do braço em torno do cotovelo e joelho, e tornozelo, bem como visto neste projeto.

Figura 1 - Prótese Mecânica da perna

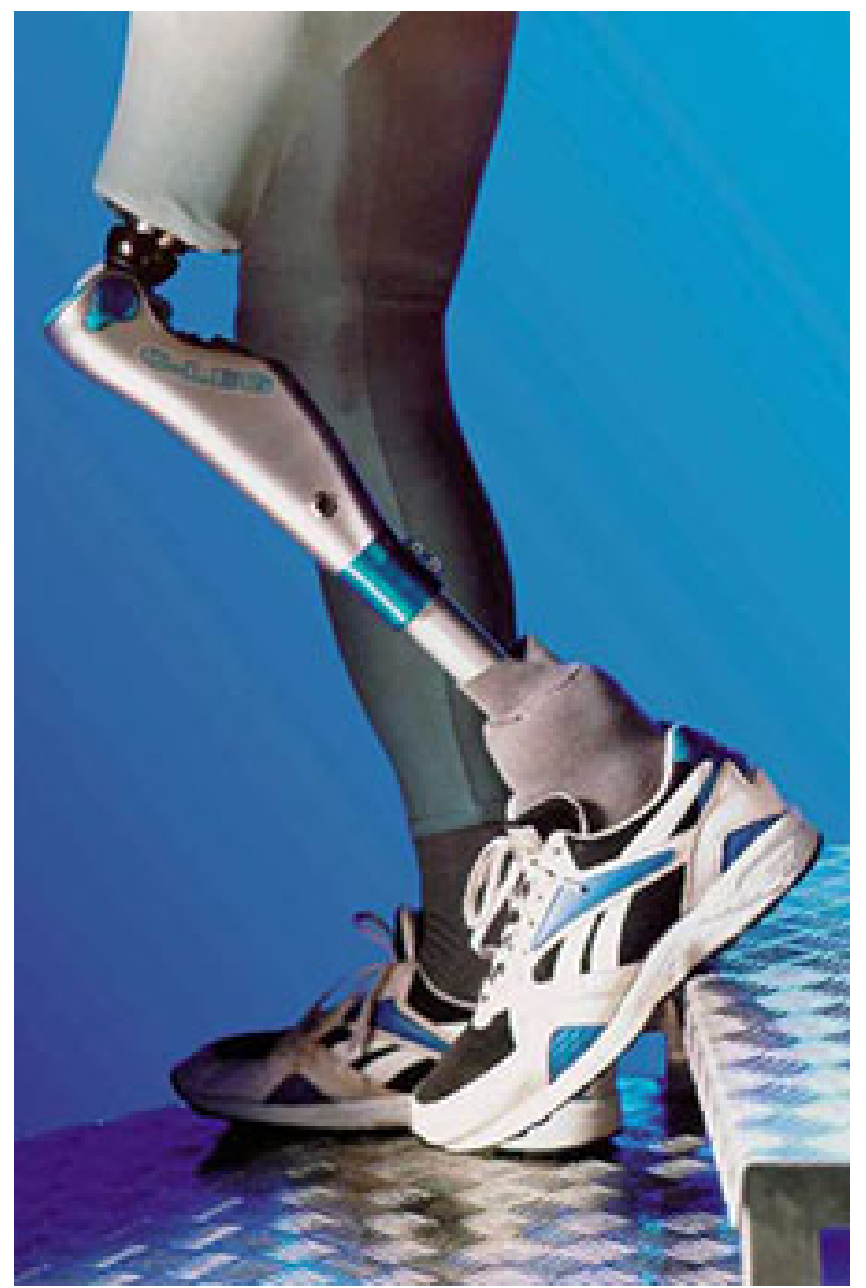


Figura 2 - Prótese Mecânica do braço - DARPA

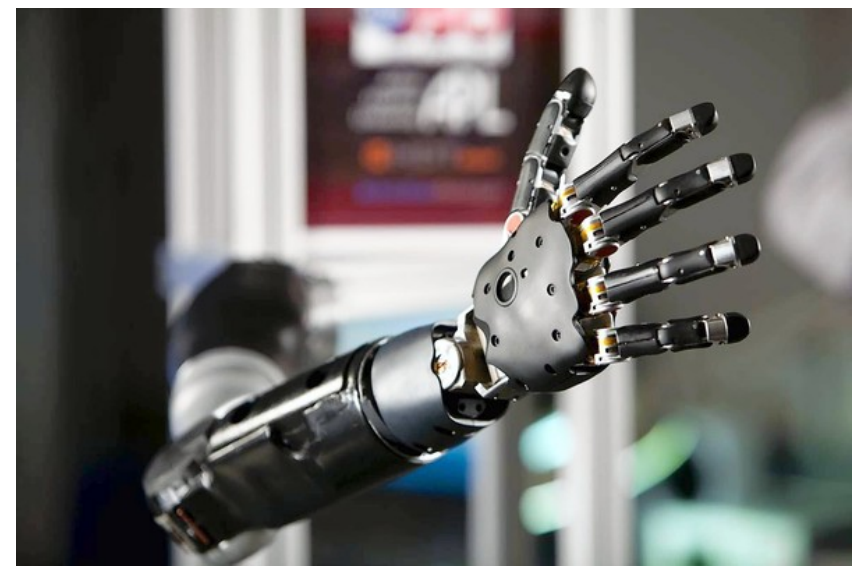

Figura 3 - Homem com prótese com precisão de segurar um ovo

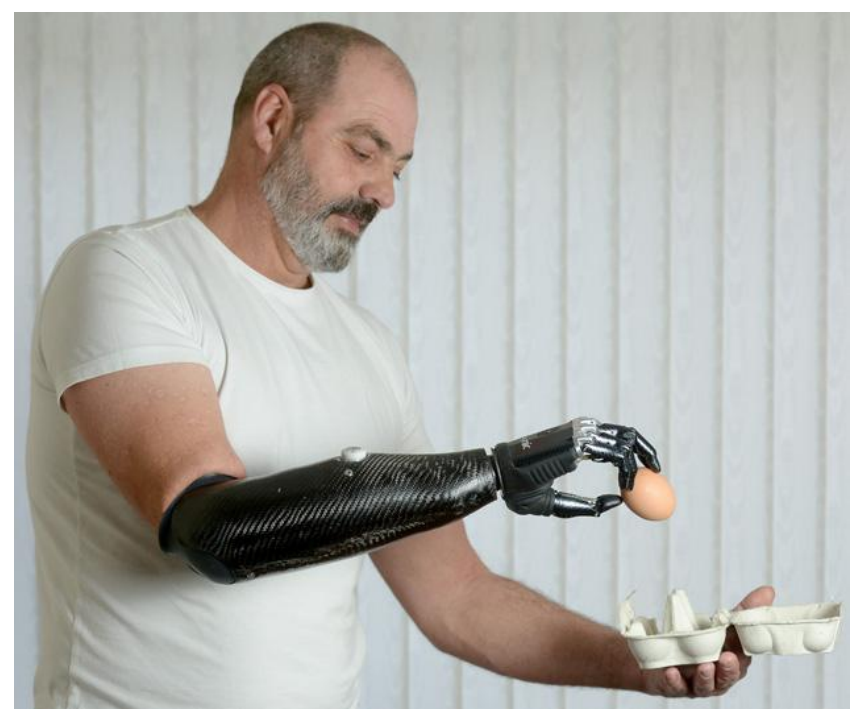

\section{Motivação}

Devido ao grande avanço da tecnologia, nos últimos 10 anos, em áreas de energia, sensoriamento, microprocessadores e microcontroladores, foi possível o desenvolvimento de próteses ativas , ou seja, aquelas que pretendem assemelhar-se às funções do membro humano. O modelo mais complexo atualmente é a prótese controlada a partir do sistema nervoso da pessoa que a utiliza.

O uso de próteses está se tornando mais público, seja pelo desenvolvimento de tecnologias de ponta, que permitem uma cópia de membros humanos, com o mesmo rendimento e por vezes até melhor; ou pelo crescente incentivo aos Jogos Paralímpicos; ou pelo barateamento das próteses mais simples, tornando-se mais acessíveis ao público em geral.

No âmbito do estudo e desenvolvimento de uma prótese mecânica ativa, inicialmente, por razões de semelhança e grau de complexidade, será projetado um modelo simplificado, baseando na pinça mecânica. Apesar da pinça ser bastante simples, com apenas um grau de liberdade de movimentação, ela se assemelha aos movimentos básicos nas juntas e articulações, onde ocorre o movimento dos braços. 


\section{Objetivo}

O projeto visa o estudo da pinça mecânica, nos aspectos mecânico, elementos mecânicos para possibilitar os movimentos; sensorial, com objetivo de assemelhar-se aos sensores do corpo humano; e de controle, o sinal recebido para os elementos mecânicos atuarem e também o feedback para informar as características da pinça no instante. O movimento a ser realizado é de abrir e fechar as garras com um objeto entre elas e analisar as forças ali captadas.

A pinça mecânica é o modelo mais básico no estudo de próteses, mas possui os conceitos básicos dos movimentos realizados por uma prótese mecânica mais complexa como um braço, por exemplo. A complexidade aumenta pela introdução de mais graus de liberdade para o movimento de um membro, refletindo nos dispositivos mecânicos utilizados e o controle requerido.

\section{Metodologia}

O estudo consiste em modelar as três principais áreas de relevância, a citar , o sensoriamento, o controle e o acionamento. A movimentação da pinça se dá por motores acoplados, a parte de sensoriamento é feito pela instalação de sensores de pressão na ponta da pinça, geralmente usados os extensômeros piezoresistivos, no qual uma deformação física do extensômero causa uma variação na resistência e uma diferença de potencial. O modelo deve expresso pelas equações que regem o movimento e, utilizando como ferramenta o MatLab, efetuar a simulação e o controle do sistema, para menor impacto mecânico no contato com o objeto.

\section{Modelagem e equacionamento}

O projeto consiste de um motor DC, duas barras que formam a pinça e o objeto a ser segurado.

O objeto tratado assume-se como uma esfera e apresentando, na região de toque, uma rigidez elástica e amortecimento.

As barras são consideradas corpos rígidos e sua elasticidade e amortecimento são integrados naquelas da esfera. Além disso, é considerada apenas uma barra conectada diretamente ao motor e portanto apenas ela possui movimento de rotação, a outra permanecendo parada encostada no objeto.

O motor DC modelado envolve um rotor acionado pela parte elétrica a partir de uma voltagem no sistema. Ambas dinâmicas elétricas e mecânicas do motor são consideradas.

As figuras abaixo representam o modelo estudado, o sistema mecânico completo e o sistema elétrico, respectivamente. 
Figura 4 - Diagrama do sistema mecânico

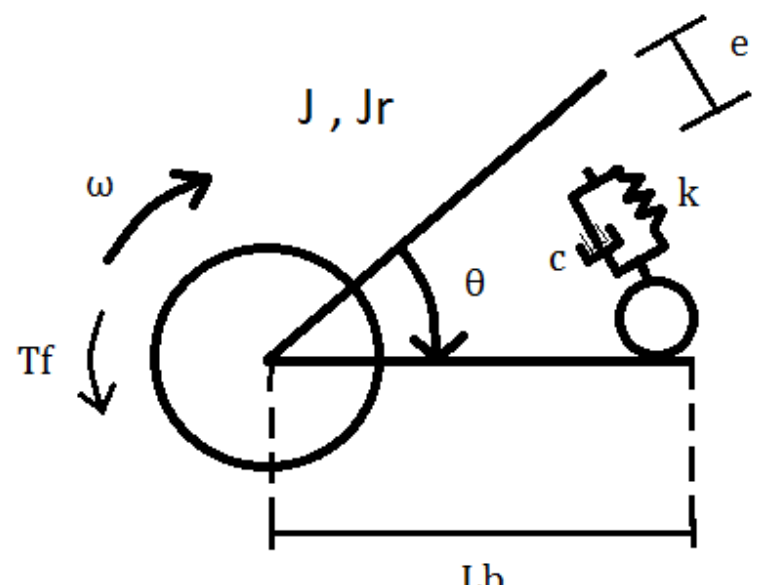

Figura 5 - Diagrama do sistema elétrico

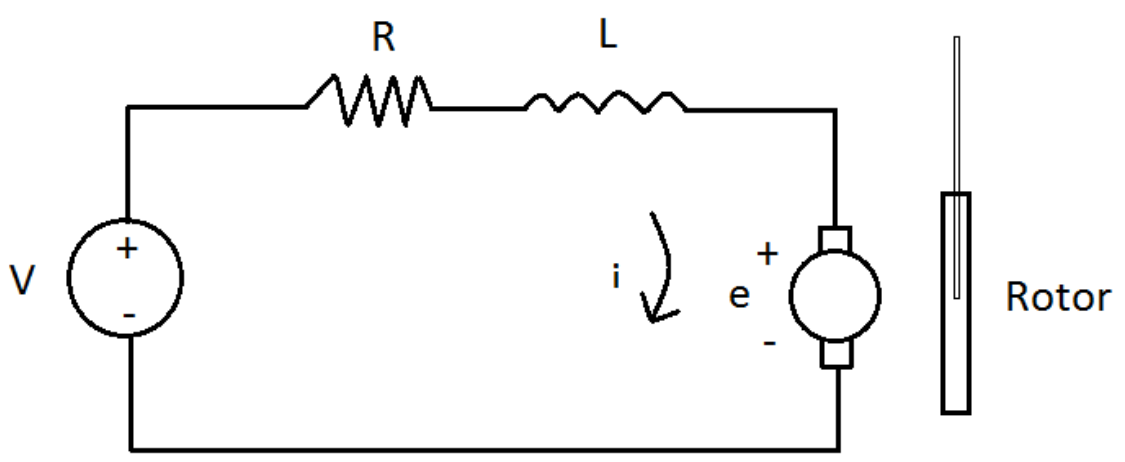

A partir dos diagramas foram escritas as duas equações que regem o sistema.

A análise elétrica é uma relação direta entre todas as grandezas envolvidas:

$\frac{d i}{d t}=\frac{-R i+V-K_{e} \dot{\theta}}{L}$.

Sendo:

- i : corrente elétrica

- $\mathrm{R}$ : resistência elétrica

- V : diferença de potencial aplicada

- $K_{e}$ : Força eletromotriz inversa

- $\dot{\theta}$ : velocidade angular do rotor

- L : Indutância 
O equacionamento é tratado como não-linear pelo fato de existir a região de contato e a região livre. Assim a equação dinâmica deve ser dividida em duas partes, na região de atuação livre, onde não há contato entre a pinça e a esfera e portanto há apenas a ação do motor elétrico e a inércia da barra,

$\ddot{\theta}=\frac{-T_{f}+K_{t} i}{J+J_{r}}$

E a região onde está segurando a esfera, surgindo forças elásticas e dissipativas provenientes da esfera,

$\ddot{\theta}=\frac{k e L-b L_{b} \dot{\theta}-T_{f}+K_{t} i}{J+J_{r}}$

Sendo:

- i : corrente elétrica.

- $\mathrm{k}$ : constante de rigidez da mola .

- e : distância entre a barra móvel e extremidade não deformada da esfera .

- $L_{b}$ : comprimento da barra .

- b : constante de amortecimento.

- $\theta$ : posição angular da barra móvel.

- $T_{f}$ : torque de atrito.

- $K_{t}$ : Sensitividade do torque, relação entre corrente elétrica e torque produzido.

- J : momento de inércia da barra.

- $J_{r}$ : momento de inércia do motor.

A variável a ser analisada para determinação da região atuante é a distância 'e', que ao se tornar menor ou igual a zero indicando que ocorreu contato e outra equação rege o sistema.

\section{Dados numéricos}

Para o modelo numérico foi escolhido um modelo real de um motor DC, com especificação de torque compatível com o projeto.

O motor escolhido é o C23-L33 W20 M00 da Moog Components Group, conforme especificado no apêndice. Para a modelagem os dados relevantes são:

- $\mathrm{R}=1 \Omega$

- $\mathrm{L}=9.4 \cdot 10^{-4} H$

- $J_{r}=1.55 \cdot 10^{-5} \mathrm{kgm}^{2}$

- $K_{t}=3 \cdot 10^{-2} \mathrm{Nm} / \mathrm{A}$

- $T_{f}=2 \cdot 10^{-2} \mathrm{Nm}$

- $K_{e}=3.01 \cdot 10^{-2} \mathrm{~V} / \mathrm{rad} / \mathrm{s}$ 
A barra que forma a pinça foi projetada como um material não especificado, variando de aplicação para aplicação. Para a simulação foi utilizada uma barra com as seguintes especificações:

- $\mathrm{m}=2 \cdot 10^{-1} \mathrm{~kg}$

- $\mathrm{l}=1.5 \cdot 10^{-1} \mathrm{~m}$

Com esses dados o momento de inércia da barra é calculado:

$J=\frac{m l^{2}}{3}$

e então $\mathrm{J}=1.5 \cdot 10^{-3} \mathrm{kgm}{ }^{2}$.

As esfera também foi modelada sem um material específico, utilizando constantes de baixa dissipação e maior rigidez, simulando um material frágil,

- $\mathrm{k}=100 \mathrm{~N} / \mathrm{m}$

- $\mathrm{b}=0.05 \mathrm{Ns} / \mathrm{m}$

\section{Modelagem em MATLAB}

Com os dados numéricos e o modelo matemático do sistema, foram feitos dois modelos no MATLAB, um em linha de comando para a simulação do efeito contato/não contato e outro em Simulink para realizar o controle do sistema na situação de contato com a esfera.

\subsection{Linha de comando}

A simulação foi realizada introduzindo as duas equações que regem o sistema e resolvendo-as pelo método de integração ode45. Após a resolução, foram calculadas as forças de toque para cada instante de tempo. Ao final, são gerados os gráficos resultantes da simulação.

As condições iniciais para a simulação foram :

$i_{0}=0$ (corrente elétrica inicial),

$\theta_{0}=-\pi / 4$ (posição angular inicial),

$\dot{\theta}_{0}=0$ (velocidade angular inicial) .

O código utilizado é o seguinte: 
Figura 6 - Código em linha de comando

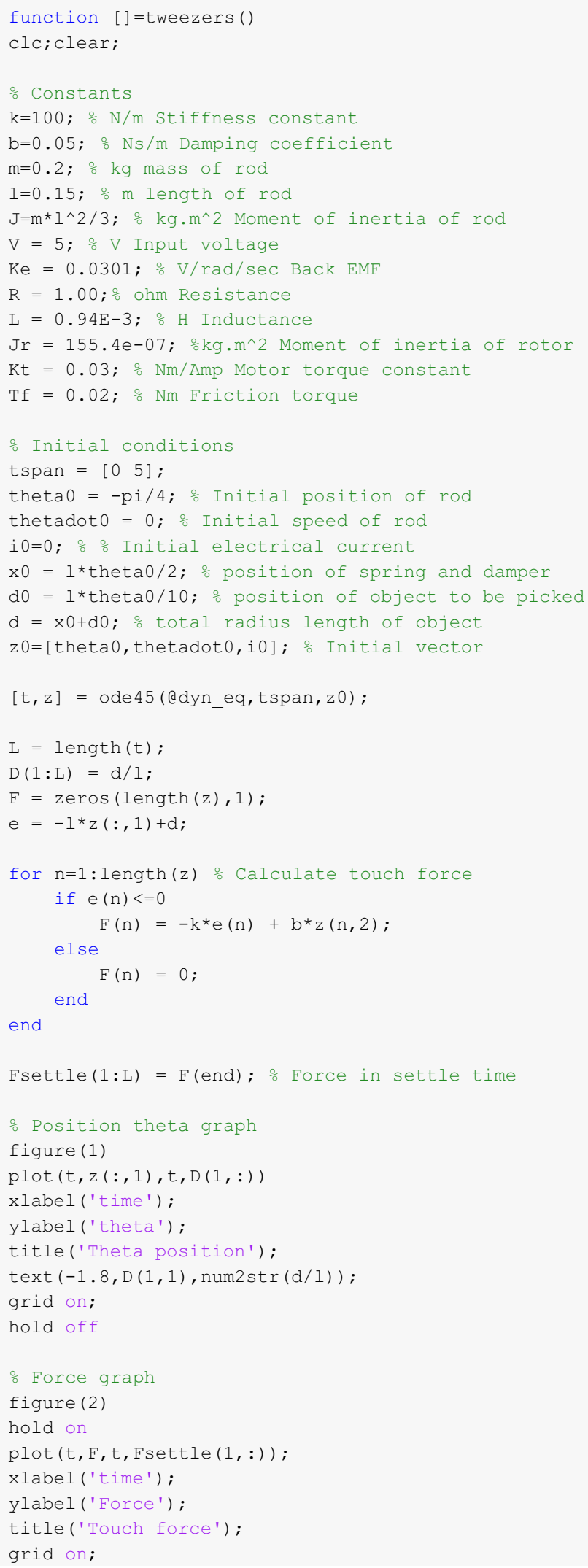




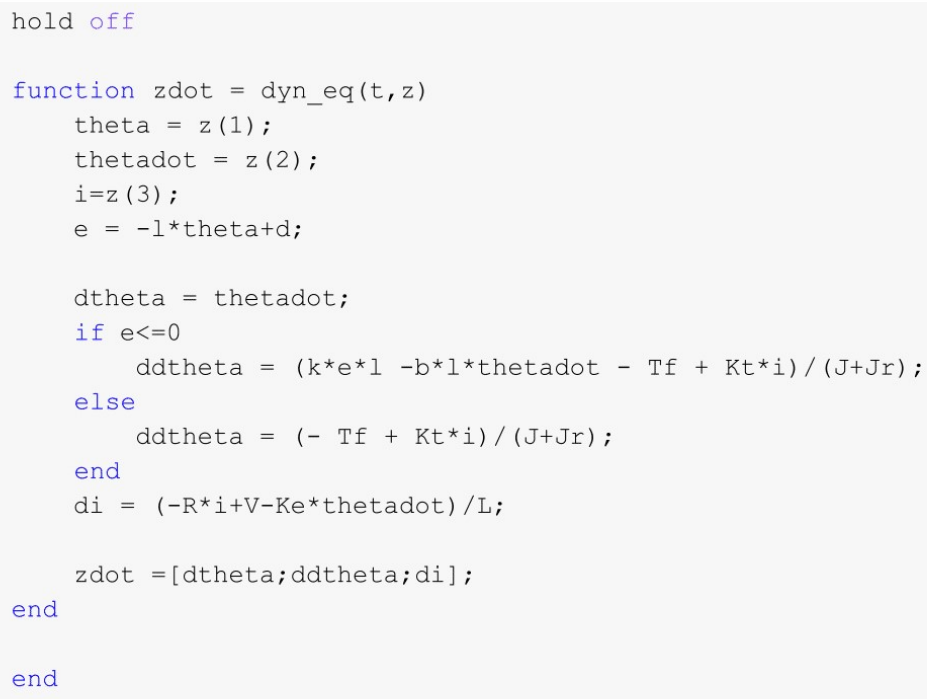

\subsection{Simulink}

No Simulink, por meio de diagramas de blocos foi montada a malha fechada de controle do sistema. No âmbito do controle a parte na qual não há toque com a esfera pode ser desconsiderada, pois o motor deve fornecer torque suficiente para a pinça ter contato, instante no qual deve-se iniciar a ação do controlador. Posto tal cenário, as condições iniciais devem ser diferentes daquelas da linha de comando, sendo:

$i_{0}=0$ (corrente elétrica inicial),

$\theta_{0}=-3 \pi / 20$ (posição angular inicial),

$\dot{\theta}_{0}=1.9 \mathrm{rad} / \mathrm{s}$ (velocidade angular inicial).

É importante notar que a velocidade angular inicial não é nula nessa simulação, pois no movimento completo do sistema, a pinça possui velocidade no instante de contato com a esfera, sendo o valor inicial utilizado a velocidade da simulação completa no instante de contato. A posição angular usada é aquela na qual há contato entre a esfera e a pinça, porém não há forças agindo nesta interface.

A variável de interesse dada como referência é a força aplicada pela pinça, pois a força indica se o torque aplicado é suficiente para segurar, se houve torque em excesso causando a quebra do objeto, ou se ele está adequado para a situação. Outra variável importante para análise é a posição angular $\theta$, de modo a saber como se comporta o movimento do sistema e o quanto a esfera é deformada ao final do movimento. Os diagramas de blocos foram estruturados, como a seguir: 
Figura 7 - Diagrama de blocos do sistema de controle

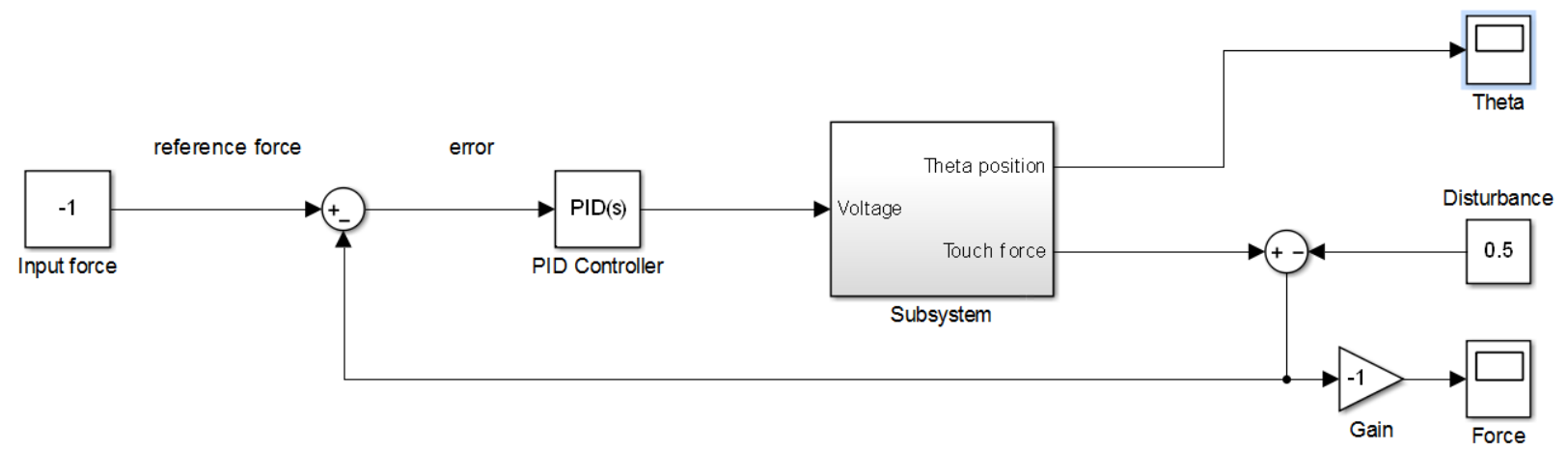

A entrada do sistema é a força de referência, na qual se deseja que o sistema estabilize e existe ainda um distúrbio no sistema que afeta a força na esfera. A saída de realimentação é a força após o controle e dinâmica. Adicionalmente, foi adicionado um distúrbio de força no sistema. A força de entrada é posta como negativa por se tratar de força de compressão, o gráfico da força é apresentado em módulo para facilitar a visualização.

Figura 8 - Diagrama de blocos das equações do sistema

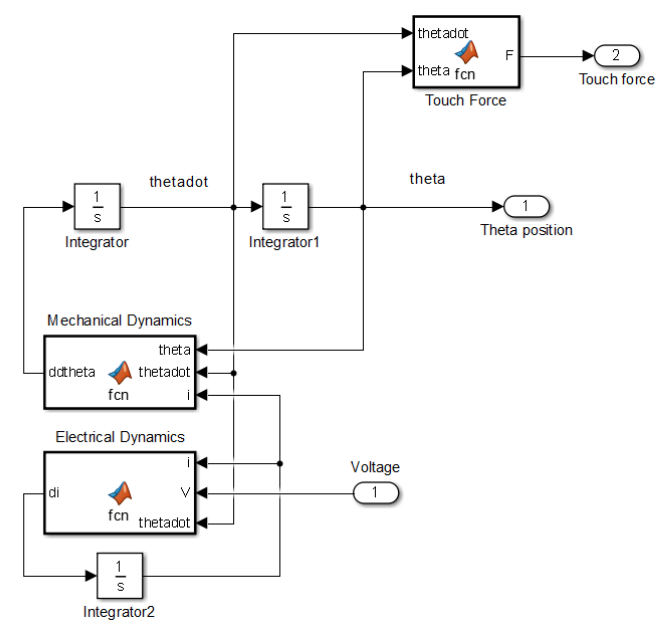

Na figura anterior , está apresentado a dinâmica do sistema e a força de contato. As equações dinâmicas foram introduzidas nos blocos de função MATLAB.

\section{Resultados}

\subsection{Linha de comando}

A simulação do sistema apresentou o seguinte para a posição angular $\theta$ em função do tempo: 
Figura 9 - Gráfico de $\theta$ por linha de comando

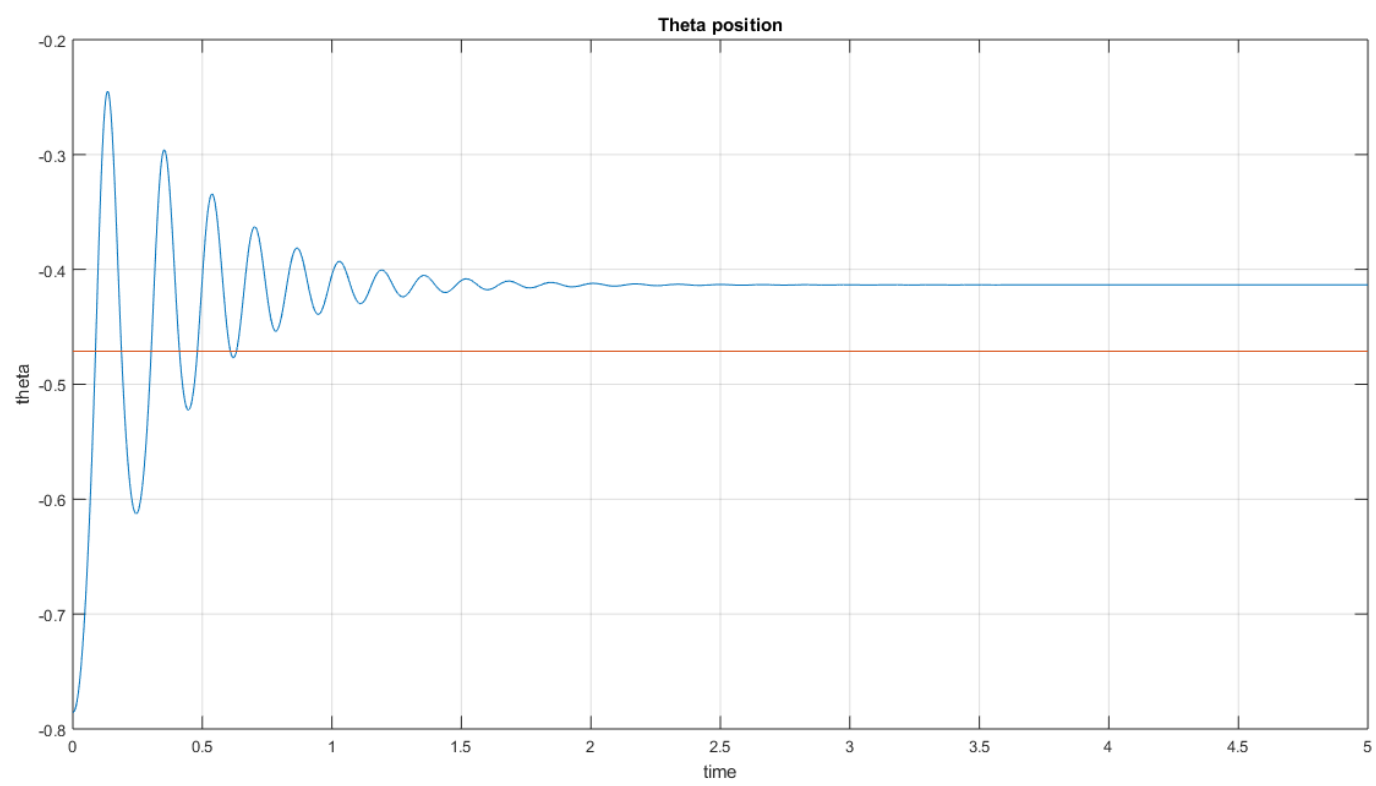

A linha vermelha no gráfico representa a posição da extremidade da esfera não deformada, ou seja toda região na qual o gráfico de $\theta$ está acima da linha representa que a esfera está deformada e consequentemente sob esforço de compressão.

O sistema apresenta um sobressinal de $68.85 \%$. Se o material do objeto suporta apenas pequenas deformações, o sobressinal ocorrido no início da simulação é suficiente para quebrar o material.

Figura 10 - Gráfico da força por linha de comando

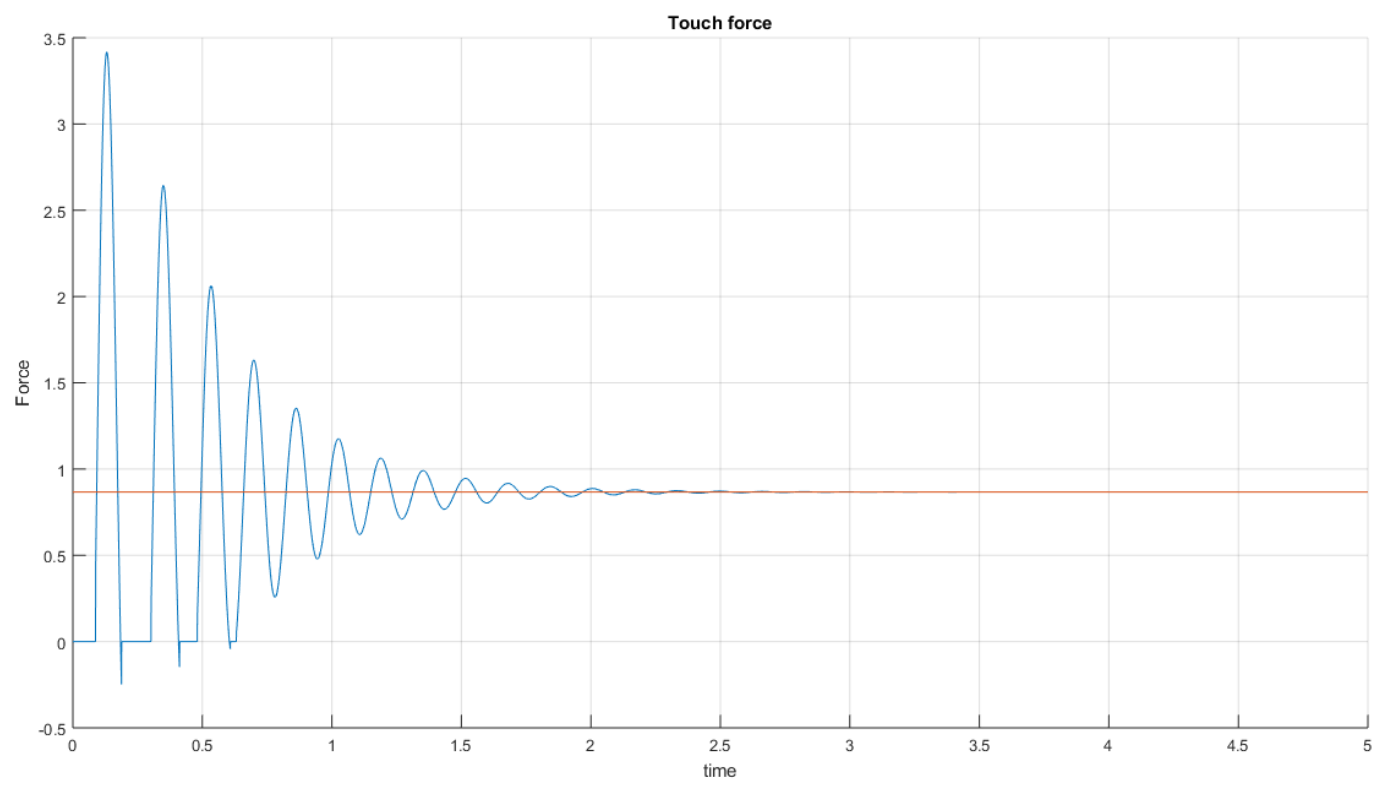

Para a força de toque, o sobressinal é ainda maior, de $294.20 \%$. O pico de força do sistema é suficiente para romper a esfera, se feita de um material de baixa resistência. A resposta obtida 
para a força mostra a necessidade de um sistema de controle, tomando como referência a força, que é a variável relevante ao se tratar de resistência de materiais.

\subsection{Simulink}

O modelo feito em Simulink apresentou resultados próximos ao desejado do mecanismo, um sistema com baixo sobressinal na posição angular e na força.

Figura 11 - Gráfico de $\theta$ por diagrama de blocos

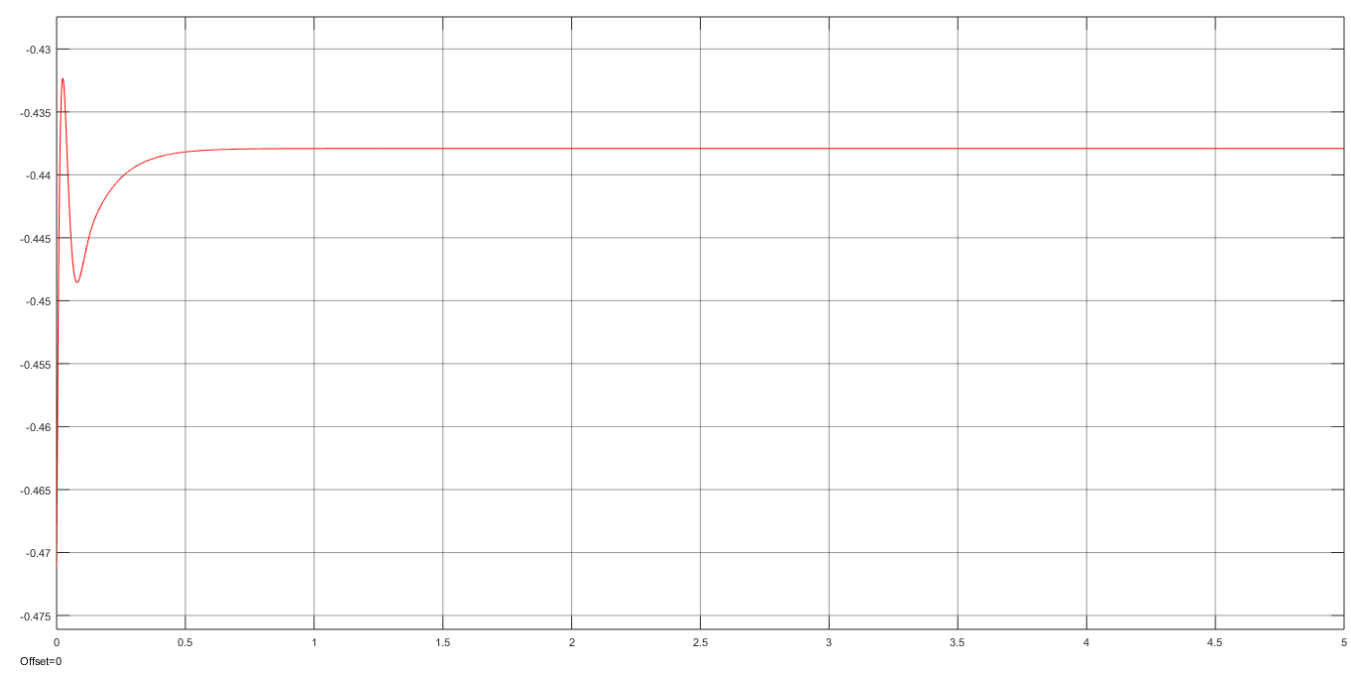

O sobressinal apresentado na posição angular foi de 1.3\%, aproximadamente 53 vezes menor do que do sistema sem controlador.

Figura 12 - Gráfico da força por diagrama de blocos

\begin{tabular}{|l|l|l|l|l|l|l|l|l|}
\hline & & & & & & & & \\
\hline
\end{tabular}

O sobressinal apresentado na força foi de $9.3 \%$, aproximadamente 31.6 vezes menor do que do sistema sem controlador.

Os ganhos no controlador PID através da ferramenta PID Tuner do MATLAB, no qual especificou-se um sistema de baixo sobressinal e tempo de estabilização menor do que 5 segundos. Os ganhos calculados foram:

$\operatorname{Proporcional}(\mathrm{P})=14.43$

$\operatorname{Integral}(\mathrm{I})=142.67$ 
$\operatorname{Derivativo}(\mathrm{D})=0.355$

Coeficiente do filtro(N) $=14683.52$

Com formula final de ganho:

$P+I \frac{1}{s}+\frac{N}{1+N \frac{1}{s}}$

\section{Conclusão}

O projeto de uma pinça mecânica com controle de força no contato com um objeto é base para todos sistemas que contenham o conceito de barras modeladas com interação com outros corpos, como braços robóticos de precisão ou próteses robóticas. A modelagem do sistema envolveu o equacionamento mecânico e elétrico de todas as partes, usando dados numéricos genéricos para a pinça e o objeto, permitindo a simulação para diversos materiais e dimensões; e um motor DC especificado adequado para pequenos torques, como simulado no projeto. O controle PID adequou os resultados ao objetivo proposto.

O projeto serve de base para projetos mais complexos, envolvendo um controle mais fino para materiais extremamente frágeis ou situações de precisão. O projeto pode ser estendido modelando as duas barras da pinça como partes móveis; a resistência mecânica do objeto, que considera o material e a geometria, obtida em geral através de métodos experimentais; e também a utilização de vários sistemas de pinça integrados, como um braço robótico completo. 


\title{
REFERÊNCIAS
}

DARPA Helps Paralyzed Man Feel Again Using a Brain-Controlled Robotic Arm(2016). Retirado de https://www.darpa.mil/news-events/2016-10-13, acessado em 31/07/2017

"No. 1705: A 3000-Year-Old Toe"Uh.edu. 2004-08-01. Acessado em 13/03/2017

Neural Signal Processing: Tutorial 1(2008). Retirado de http://www-users.med.cornell.edu/ jdvicto/pdfs/pubo08.pdf, acessado em 01/08/2017

Permanent Magnet DC Motors. Retirado de http://www.moog.com/literature/MCG/moc23series.pdf, acessado em 26/07/2017

Ogata, K. Engenharia de Controle Moderno. 5 a ed., 2010

\section{Title - Modeling and simulation of sensory mechanical tweezer}

\begin{abstract}
The project is based upon the modeling, simulation and control, executed in the MATLAB software, of mechanical tweezers driven by a DC motor, with the objective of holding an object of low mechanical resistance to superficial pressures, requiring a precise control under the applied force in the object. The project is the study of a simple model of one single degree of freedom, but it is the base to current mechanical prosthesis models, with more degrees of freedom.
\end{abstract}

Keywords - Modeling, Control, Force, Degree of freedom, Prosthesis

Marcos Felipe Saito, Aluno de Engenharia Mecatrônica na Escola Politécnica da Universidade de São Paulo e aluno de Engenharia Mecatrônica da CentraleSupélec(França). Ex-monitor de PME-3100 e PME-3200 na Escola Politécnica da Universidade de São Paulo. Aluno de graduação no Laboratório de Dinâmica e Simulação Veicular.

\section{ANEXO A - Especificações do motor DC magnético permanente}




\section{TYPICAL APPLICATIONS}

Robotics and factory automation

- Pick-and-place robots

- Positioning tables

- Welding wire feeders

- Automatic guided vehicles

- Barcoding equipment

Computer and office equipment

- Copier and microfilm machines

- Printers / plotters

- Tape drives

Industrial equipment

- Automatic door actuators

- Material handling equipment

- Packaging, marking and sorting equipment

- Machine tools

- Web drives

- Gimbal controlled cameras for security systems

- Antenna drives

Medical equipment

- Electric wheelchairs and scooters

- Bio-analytical equipment

- Medical pumps

- Centrifuges

\section{FEATURES}

- Long-life, externally replaceable brushes; various grade materials available for high / low voltage applications

- Superior protection provided by totally enclosed, high strength, zinc-plated steel housing

- Shaft configuration optional

- Machined aluminum end-cap for precise locating; round or square. Precision-tapped mounting holes provided to your specifications

- Silicon steel laminations

- Diamond turned commutator for quiet operation and long brush life

- Skewed rotors available for minimal cogging torque

- Rotors are dynamically balanced to ISO G2.5

- Available with standard NEMA mountings

- Polyester resin impregnated insulated windings

- Double-shielded, permanently lubricated ball bearings, ABEC 5 standard; others optional

\section{BENEFITS}

- Optional pre-aligned encoders provide accurate positioning

- Tachometers are available - 7, 10, 14 V / KRPM

- These motors offer continuous torques from 16.5 to 560 oz-in, peak torques from 125 to 3500 oz-in

- Motor lengths -3.33 to 9.0 inches

- Diameter - 2.25 to 4.0 inches

- Permanently lubricated bearings

- Available with carbon steel or stainless steel shafts; single or double ended extensions

- Custom shaft and end cap configurations are also available

\section{Permanent Magnet DC Motors}

\section{C23, 34, 42 Series}

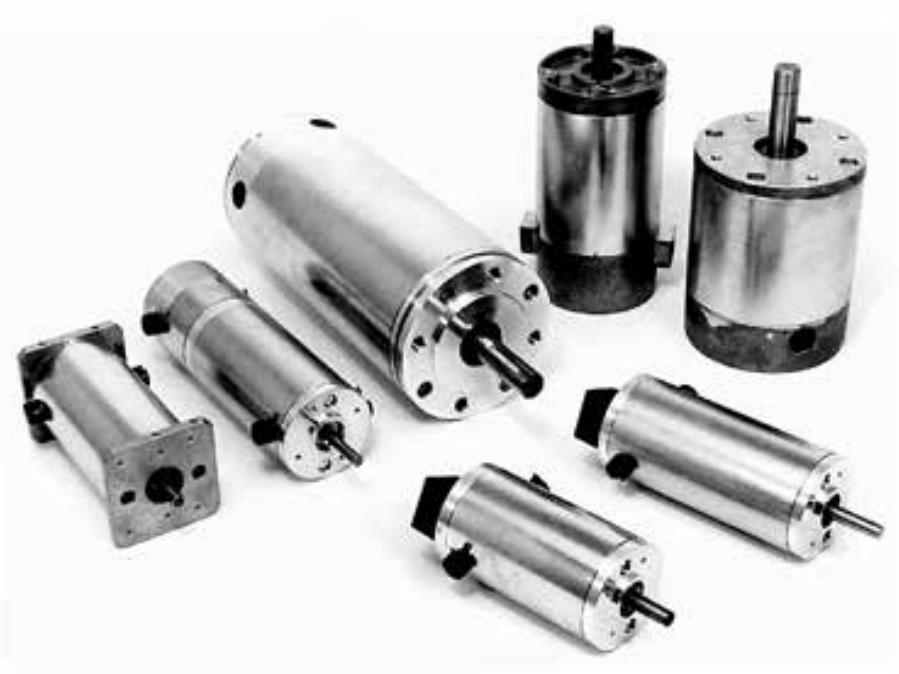

\section{Available with integrated tachometers, resolvers and encoders for closed-loop control}

Moog Components Group offers a complete line of 2.25 to 4 inches diameter permanent magnet motors. Integrated feedback devices (tachometers and encoders) are available for closed-loop control.

We offer a variety of standard sizes. If mechanical modifications are needed, custom options are available for your specific application. Our engineering department is prepared to discuss your application to help tailor a permanent magnet motor to fit your needs.

Note: This catalog contains basic marketing information and general part descriptions of Moog

Components Group product lines. With respect to the U.S. export regulations, the products described herein are controlled by the U.S. Commerce Department or the U.S. State Department. Contact Moog Components Group for additional detail on the export controls that are applicable to your part. 


\section{C23 Series Speciffications}

\section{SPECIFICATION AND NUMBERING SYSTEM}

Part Numbering System
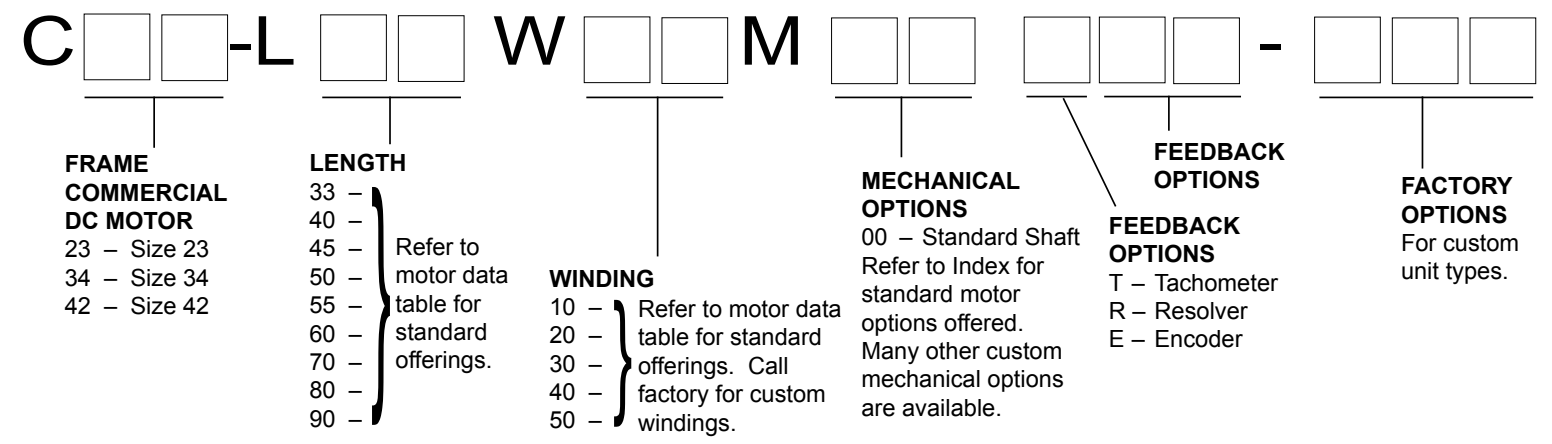

C23 SERIES SPECIFICATIONS - Continuous Stall Torque 16.5 - 27 oz-in (0.117 - 0.191 Nm) Peak Torque 125 - 250 oz-in (0.883 - 1.765 Nm)

\begin{tabular}{|c|c|c|c|c|c|c|c|c|c|c|c|}
\hline \multicolumn{2}{|c|}{ Part Number* } & \multicolumn{5}{|c|}{ C23-L33 } & \multicolumn{5}{|c|}{ C23-L40 } \\
\hline Winding Code ${ }^{* *}$ & & 10 & 20 & 30 & 40 & 50 & 10 & 20 & 30 & 40 & 50 \\
\hline \multirow[t]{2}{*}{$L=$ Length } & inches & \multicolumn{5}{|c|}{3.33} & \multicolumn{5}{|c|}{4} \\
\hline & millimeters & \multicolumn{5}{|c|}{84.6} & \multicolumn{5}{|c|}{101.6} \\
\hline \multirow[t]{2}{*}{ Peak Torque } & oz-in & 125.0 & 125.0 & 125.0 & 125.0 & 125.0 & 250.0 & 250.0 & 250.0 & 250.0 & 250.0 \\
\hline & $\mathrm{Nm}$ & 0.883 & 0.883 & 0.883 & 0.883 & 0.883 & 1.765 & 1.765 & 1.765 & 1.765 & 1.765 \\
\hline \multirow[t]{2}{*}{ Continuous Stall Torque } & oz-in & 16.5 & 16.5 & 16.5 & 16.5 & 16.5 & 27.0 & 27.0 & 27.0 & 27.0 & 27.0 \\
\hline & $\mathrm{Nm}$ & 0.117 & 0.117 & 0.117 & 0.117 & 0.117 & 0.191 & 0.191 & 0.191 & 0.191 & 0.191 \\
\hline Rated Terminal Voltage & volts DC & $12-24$ & $12-24$ & $12-36$ & $12-60$ & $12-60$ & $12-24$ & $12-48$ & $12-60$ & $12-60$ & $12-60$ \\
\hline Terminal Voltage & volts $\mathrm{DC}$ & 12 & 12 & 24 & 36 & 48 & 12 & 24 & 36 & 48 & 60 \\
\hline \multirow[t]{2}{*}{ Rated Speed } & RPM & 4700 & 2150 & 4200 & 3750 & 3000 & 2300 & 3600 & 3500 & 2850 & 2250 \\
\hline & $\mathrm{rad} / \mathrm{sec}$ & 492 & 225 & 440 & 393 & 314 & 241 & 377 & 367 & 298 & 236 \\
\hline \multirow[t]{2}{*}{ Rated Torque } & oz-in & 7.5 & 12.6 & 12.7 & 14.4 & 15.8 & 17.3 & 25.5 & 25.3 & 25.6 & 24.2 \\
\hline & $\mathrm{Nm}$ & 0.05 & 0.09 & 0.09 & 0.10 & 0.11 & 0.12 & 0.18 & 0.18 & 0.18 & 0.17 \\
\hline Rated Current & Amps & 4.75 & 4.3 & 3 & 2 & 1.4 & 4.9 & 4.3 & 2.75 & 1.8 & 1.1 \\
\hline \multirow[t]{2}{*}{ Rated Power } & Watts & 26.1 & 20.0 & 39.5 & 40.0 & 35.1 & 29.4 & 67.9 & 65.5 & 54.0 & 40.3 \\
\hline & Horsepower & 0.03 & 0.03 & 0.05 & 0.05 & 0.05 & 0.04 & 0.09 & 0.09 & 0.07 & 0.05 \\
\hline \multirow[t]{2}{*}{ Torque Senstivity } & oz-in/amp & 2.65 & 4.25 & 6.2 & 10.25 & 15.75 & 4.84 & 7.74 & 12 & 18.5 & 28.75 \\
\hline & Nm/amp & 0.0187 & 0.0300 & 0.0438 & 0.0724 & 0.1112 & 0.0342 & 0.0547 & 0.0847 & 0.1306 & 0.2030 \\
\hline \multirow[t]{2}{*}{ Back EMF } & volts/KRPM & 2 & 3.15 & 4.6 & 7.6 & 11.5 & 3.58 & 5.72 & 8.82 & 13.82 & 21.22 \\
\hline & volts/rad/sec & 0.0191 & 0.0301 & 0.0439 & 0.0726 & 0.1098 & 0.0342 & 0.0546 & 0.0842 & 0.1320 & 0.2026 \\
\hline Terminal Resistance & ohms & 0.60 & 1.00 & 1.70 & 4.00 & 9.00 & 0.70 & 0.96 & 2.30 & 5.50 & 12.00 \\
\hline Terminal Inductance & $\mathrm{mH}$ & 0.35 & 0.94 & 2.00 & 5.50 & 13.00 & 0.50 & 1.30 & 3.10 & 7.36 & 18.00 \\
\hline \multirow[t]{2}{*}{ Motor Constant } & oz-in/watt ${ }^{\wedge} 1 / 2$ & 3.4 & 4.3 & 4.8 & 5.1 & 5.3 & 5.8 & 7.9 & 7.9 & 7.9 & 8.3 \\
\hline & $\mathrm{Nm} /$ watt & 0.024 & 0.030 & 0.034 & 0.036 & 0.037 & 0.041 & 0.056 & 0.056 & 0.056 & 0.059 \\
\hline \multirow[t]{2}{*}{ Rotor Inertia } & oz-in-sec ${ }^{2}$ & 0.0022 & 0.0022 & 0.0022 & 0.0022 & 0.0022 & 0.004 & 0.004 & 0.004 & 0.004 & 0.004 \\
\hline & $\mathrm{g}-\mathrm{cm}^{2}$ & 155.4 & 155.4 & 155.4 & 155.4 & 155.4 & 282.5 & 282.5 & 282.5 & 282.5 & 282.5 \\
\hline \multirow[t]{2}{*}{ Friction Torque } & oz-in & 3.0 & 3.0 & 3.0 & 3.0 & 3.0 & 3.0 & 3.0 & 3.0 & 3.0 & 3.0 \\
\hline & $\mathrm{Nm}$ & 0.02 & 0.02 & 0.02 & 0.02 & 0.02 & 0.02 & 0.02 & 0.02 & 0.02 & 0.02 \\
\hline Thermal Resistance & ${ }^{\circ} \mathrm{C} /$ watt & 6.2 & 6.2 & 6.2 & 6.2 & 6.2 & 5.4 & 5.4 & 5.4 & 5.4 & 5.4 \\
\hline \multirow[t]{2}{*}{ Damping Factor } & oz-in/KRPM & 0.1 & 0.1 & 0.1 & 0.1 & 0.1 & 0.1 & 0.1 & 0.1 & 0.1 & 0.1 \\
\hline & $\mathrm{Nm} / \mathrm{KRPM}$ & 0.001 & 0.001 & 0.001 & 0.001 & 0.001 & 0.001 & 0.001 & 0.001 & 0.001 & 0.001 \\
\hline \multirow[t]{2}{*}{ Weight } & $\mathrm{oz}$ & 27 & 27 & 27 & 27 & 27 & 38 & 38 & 38 & 38 & 38 \\
\hline & $\mathrm{g}$ & 765 & 765 & 765 & 765 & 765 & 1077 & 1077 & 1077 & 1077 & 1077 \\
\hline Electrical Time Constant & millisecond & 0.5833 & 0.9400 & 1.1765 & 1.3750 & 1.4444 & 0.7143 & 1.3584 & 1.3478 & 1.3382 & 1.5000 \\
\hline Mech. Time Constant & millisecond & 26.07623 & 17.2056 & 13.72994 & 11.82747 & 11.44547 & 16.91906 & 9.052773 & 9.100907 & 9.00927 & 8.237676 \\
\hline Speed/Torque Gradient & rpm/oz-in & -113.2075 & -74.69655 & -59.60729 & \begin{tabular}{|l|}
-51.34788 \\
\end{tabular} & -49.68944 & -40.39891 & \begin{tabular}{|l|}
-21.61598 \\
\end{tabular} & -21.73091 & -21.51211 & -19.66971 \\
\hline
\end{tabular}

Notes:

(continued on next page)

1. For MS (military style) connector, please specify connector housing and terminal.

2. Data for informational purposes only. Should not be considered a binding performance agreement. For specific applications, please contact the factory.

*Many other custom mechanical options are available - consult factory.

${ }^{* *}$ Many other winding options are available - consult factory. 


\section{C23 Series Specifications}

C23 SERIES SPECIFICATIONS -

Continuous Stall Torque 34 - 50 oz-in (0.240 - $0.353 \mathrm{Nm})$

Peak Torque 310 - 430 oz-in (2.189 - 3.037 Nm)

\begin{tabular}{|c|c|c|c|c|c|c|c|c|c|c|c|c|c|c|c|c|}
\hline \multicolumn{2}{|c|}{ Part Number* } & \multicolumn{5}{|c|}{ C23-L45 } & \multicolumn{5}{|c|}{ C23-L50 } & \multicolumn{5}{|c|}{ C23-L55 } \\
\hline Winding Code ${ }^{* *}$ & & 10 & 20 & 30 & 40 & 50 & 10 & 20 & 30 & 40 & 50 & 10 & 20 & 30 & 40 & 50 \\
\hline \multirow[t]{2}{*}{$L=$ Length } & inches & \multicolumn{5}{|c|}{4.5} & \multicolumn{5}{|c|}{5} & \multicolumn{5}{|c|}{5.45} \\
\hline & millimeters & \multicolumn{5}{|c|}{114.3} & \multicolumn{5}{|c|}{127.0} & \multicolumn{5}{|c|}{138.4} \\
\hline Peak Torque & $\mathrm{Nm}$ & 2.189 & 2.189 & 2.189 & 2.189 & 2.189 & 2.542 & 2.542 & 2.542 & 2.542 & 2.542 & 3.037 & 3.037 & 3.037 & 3.037 & 3.037 \\
\hline \multirow[t]{2}{*}{ Continuous Stall Torque } & oz-in & 34.0 & 34.0 & 34.0 & 34.0 & 34.0 & 42.0 & 42.0 & 42.0 & 42.0 & 42.0 & 50.0 & 50.0 & 50.0 & 50.0 & 50.0 \\
\hline & $\mathrm{Nm}$ & 0.240 & 0.240 & 0.240 & 0.240 & 0.240 & 0.297 & 0.297 & 0.297 & 0.297 & 0.297 & 0.353 & 0.353 & 0.353 & 0.353 & 0.353 \\
\hline \multirow[t]{2}{*}{ Rated Speed } & RPM & 1950 & 2600 & 2600 & 2100 & 1555 & 1600 & 2150 & 2150 & 1800 & 1283 & 1350 & 1800 & 1700 & 1300 & 887 \\
\hline & $\mathrm{rad} / \mathrm{sec}$ & 204 & 272 & 272 & 220 & 163 & 168 & 225 & 225 & 188 & 134 & 141 & 188 & 178 & 136 & 93 \\
\hline \multirow[t]{2}{*}{ Rated Torque } & oz-in & 25.3 & 26.5 & 25.8 & 23.3 & 23 & 27.1 & 30.1 & 32 & 31.5 & 34.3 & 36.4 & 39.3 & 40.5 & 40.9 & 43.5 \\
\hline & $\mathrm{Nm}$ & 0.18 & 0.19 & 0.18 & 0.16 & 0.16 & 0.19 & 0.21 & 0.23 & 0.22 & 0.24 & 0.26 & 0.28 & 0.29 & 0.29 & 0.31 \\
\hline Rated Current & Amps & 5.8 & 3.75 & 2.4 & 1.4 & 0.95 & 5.1 & 3.5 & 2.4 & 1.5 & 1.05 & 5.6 & 3.75 & 2.5 & 1.6 & 1.1 \\
\hline Rated Power & Watts & 36.5 & 51.0 & 49.6 & 36.2 & 26.5 & 32.1 & 47.9 & 50.9 & 42.0 & 32.6 & 36.4 & 52.3 & 50.9 & 39.3 & 28.6 \\
\hline Back EMF & volts/rad/sec & 0.0430 & 0.0688 & 0.1050 & 0.1647 & 0.2531 & 0.0517 & 0.0826 & 0.1270 & 0.1996 & 0.3056 & 0.0620 & 0.0991 & 0.1528 & 0.2401 & 0.3676 \\
\hline Terminal Resistance & ohms & 0.54 & 1.40 & 3.27 & 8.13 & 19.0 & 0.63 & 1.60 & 3.20 & 7.00 & 16.50 & 0.56 & 1.43 & 3.39 & 8.40 & 19.10 \\
\hline Terminal Inductance & $\mathrm{mH}$ & 0.72 & 1.75 & 4.26 & 10.24 & 24.20 & 0.77 & 1.96 & 4.66 & 11.44 & 27.00 & 0.97 & 2.38 & 5.50 & 13.73 & 32.28 \\
\hline \multirow[t]{2}{*}{ Motor Constant } & oz-in/watt $1 / 2$ & 8.2 & 8.2 & 8.2 & 8.2 & 8.2 & 9.3 & 9.2 & 10.1 & 10.7 & 10.7 & 11.7 & 11.7 & 11.7 & 11.7 & 11.7 \\
\hline & $\mathrm{Nm} / \mathrm{watt}$ & 0.058 & 0.058 & 0.058 & 0.058 & 0.058 & 0.065 & 0.065 & 0.071 & 0.076 & 0.075 & 0.083 & 0.083 & 0.083 & 0.083 & 0.083 \\
\hline \multirow[t]{2}{*}{ Rotor Inertia } & oz-in-sec ${ }^{2}$ & 0.0052 & 0.0052 & 0.0052 & 0.0052 & 0.0052 & 0.0065 & 0.0065 & 0.0065 & 0.0065 & 0.0065 & 0.0078 & 0.0078 & 0.0078 & 0.0078 & 0.0078 \\
\hline & g-cm² & 367.2 & 367.2 & 367.2 & 367.2 & 367.2 & 459.0 & 459.0 & 459.0 & 459.0 & 459.0 & 550.8 & 550.8 & 550.8 & 550.8 & 550.8 \\
\hline \multirow[t]{2}{*}{ Friction Torque } & oz-in & 5 & 5 & 5 & 5 & 5 & 5 & 5 & 5 & 5 & 5 & 6 & 6 & 6 & 6 & 6 \\
\hline & $\mathrm{Nm}$ & 0.04 & 0.04 & 0.04 & 0.04 & 0.04 & 0.04 & 0.04 & 0.04 & 0.04 & 0.04 & 0.04 & 0.04 & 0.04 & 0.04 & 0.04 \\
\hline Thermal Resistance & ${ }^{\circ} \mathrm{C} /$ watt & 4.7 & 4.7 & 4.7 & 4.7 & 4.7 & 4.3 & 4.3 & 4.3 & 4.3 & 4.3 & 3.9 & 3.9 & 3.9 & 3.9 & 3.9 \\
\hline \multirow[t]{2}{*}{ Damping Factor } & oz-in/KRPM & 0.2 & 0.2 & 0.2 & 0.2 & 0.2 & 0.2 & 0.2 & 0.2 & 0.2 & 0.2 & 0.3 & 0.3 & 0.3 & 0.3 & 0.3 \\
\hline & $\mathrm{Nm} / \mathrm{KRPM}$ & 0.001 & 0.001 & 0.001 & 0.001 & 0.001 & 0.001 & 0.001 & 0.001 & 0.001 & 0.001 & 0.002 & 0.002 & 0.002 & 0.002 & 0.002 \\
\hline Weight & $\mathrm{oz}$ & 46 & 46 & 46 & 46 & 46 & 56 & 56 & 56 & 56 & 56 & 65 & 65 & 65 & 65 & 65 \\
\hline
\end{tabular}

\section{C23 Typical Outline Drawing}
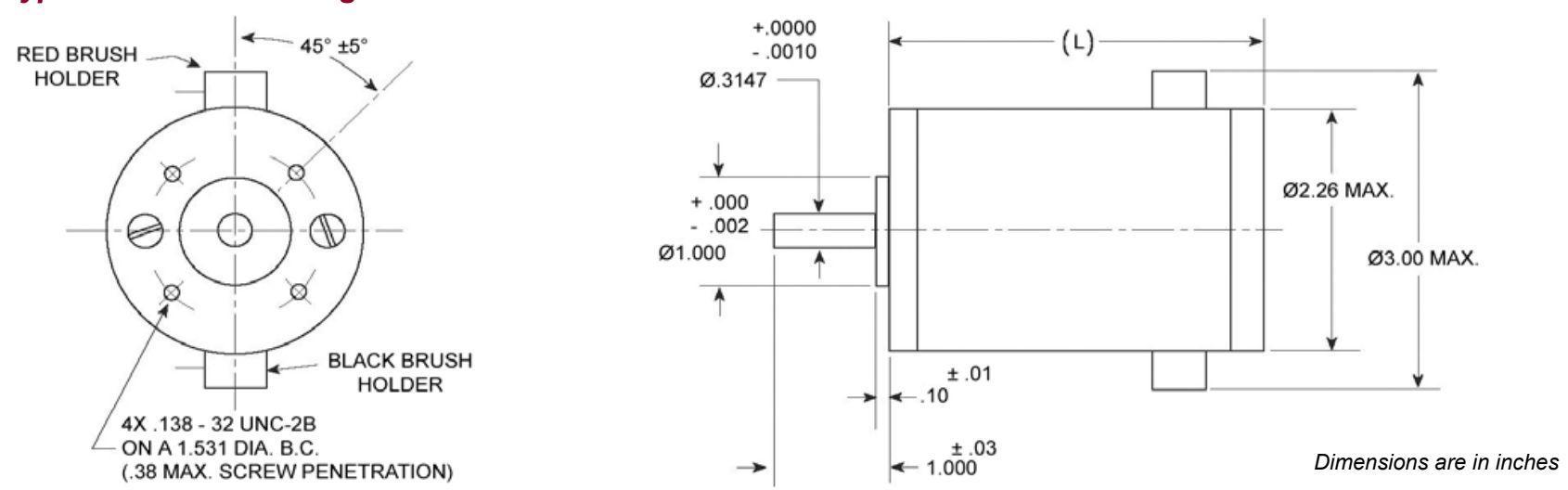

IMPORTANT

Typical performance characteristics at $25^{\circ} \mathrm{C}$. The operational life of any motor is dependent upon individual operating parameters, environment, temperature and other factors. Your specific application results may vary. Please consult the factory to discuss your requirements. 


\section{C23 Performance Curves}

\section{C23 Torque / Speed Curves}

C23-L33: 24 Volt input

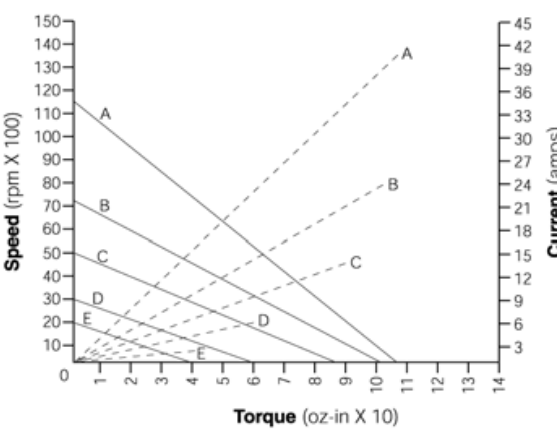

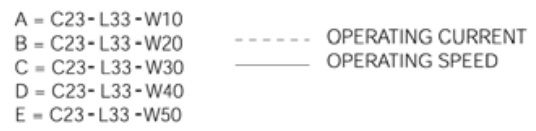

C23-L40: 24 Volt input

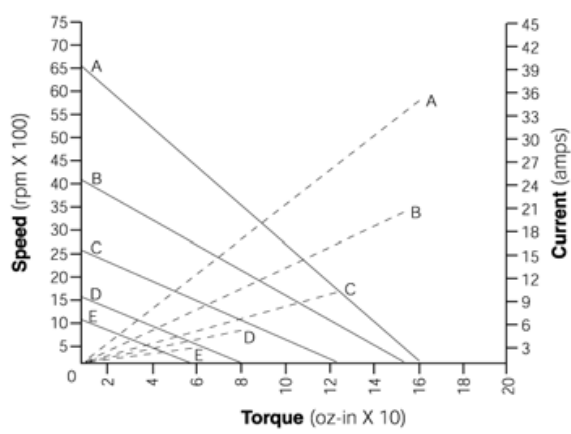
$A=C 23-L 40-W 10$
$B=C 23-L 40-W 20$
$C=C 23-L 40-W 30$
$D=C 23-L 40-W 40$
$E=C 23-L 40-W 50$
C23-L45: 24 Volt input

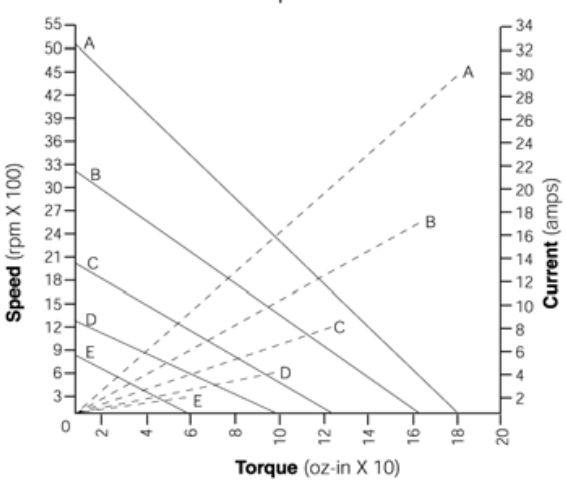

$A=C 23-L 45-W 10$

$A=C 23-L 45-W 10$
$B=C 23-L 45-W 20$

$\mathrm{B}=\mathrm{C} 23-\mathrm{L} 45-\mathrm{W} 30$

$C=C 23-L 45-W 30$
$D=C 23-L 45-W 40$

$E=C 23-L 45-W 50$
OPERATING CURRENT OPERATING SPEED
C23-L50: 24 Volt input

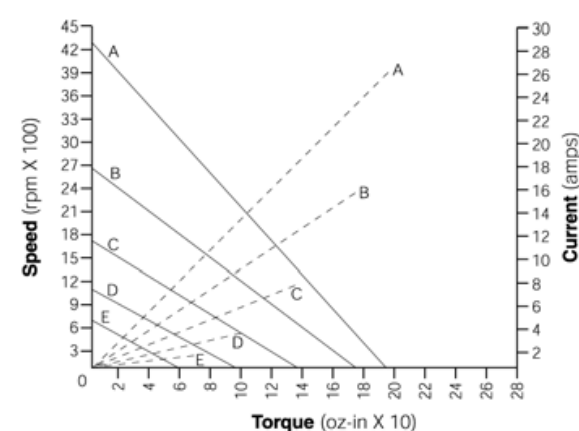

Torque (oz-in X 10)

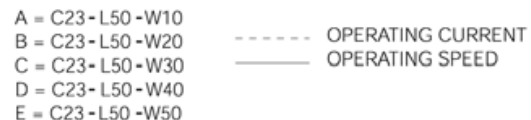

C23-L55: 36 Volt input

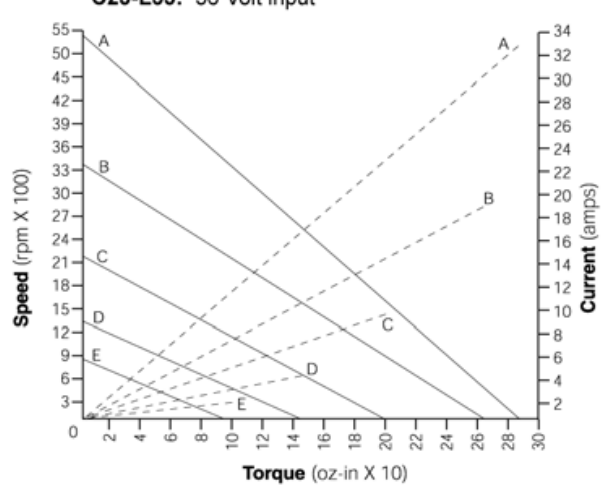

$A=C 23-L 55-W 10$

$\mathrm{B}=\mathrm{C} 23-\mathrm{L} 55-\mathrm{W} 20$ - . . . OPERATING CURREN $C=\mathrm{C} 23-\mathrm{L} 55-\mathrm{W} 30$ $\mathrm{D}=\mathrm{C} 23-\mathrm{L} 55-\mathrm{W} 40$

- Skewed Armatures standard on C13 series - available on all other series upon request.

\section{Note:}

A skewed armature is one in which the laminations do not line up, but rather are on a skew for the length of the armature stack. There are some distinct advantages to a motor utilizing a skewed armature, the greatest being a REDUCTION OF COGGING. 